\title{
Simulation of Deformation Behavior of Polymeric Materials by Chain Network Model
}

\author{
A. Shinozaki, K. Kishimoto, $\mathrm{H}$, Inoue \\ Department of Mechanical and Control Engineering, Tokyo Institute of Technology \\ 2-12-1, O-okayama, Meguro-ku, Tokyo, 152-8552, Japan
}

ABSTRACT

Recently, the electronics devices become smaller and thinner. The polymers are used for advantage of lightweight and good formability. It is important to understand the mechanical properties of polymers for design of the electronics devices. The mechanical properties of polymers are strongly influenced by meso-scale $\left(10^{-9} \sim 10^{-3} \mathrm{~m}\right)$ structure such as entanglement, molecular weight distribution, etc. However, the relationship between the meso-scale structure and macro-scale mechanical properties of poly mers has not been clarified. The difficulties plaguing the task of probing the basic mechanisms governing polymer behavior stem from the complexities of the meso-scale structure that dominates the response of the material. Some complexities, chain entanglement, slip, Van der Waals' force, make direct experimental identification of these mechanisms extremely difficult. Understanding the contribution of the meso-scale structure to the mechanical properties can provide a better basis for continuum-level modeling, as well as provide a more fundamental understanding of the connection between meso-scale structure and mechanical properties in polymeric materials. In these days, many studies about problem of properties of polymer have been made by molecular dynamics. It is possible to clear the detail atomic behaviors of polymer using by atomistic molecular dynamics $\left(10^{-10} \sim 10^{-9} \mathrm{~m}\right)$. However, it is not easy to simulate the meso-scale molecular behaviors, because of ungodly amount of computational time. In this paper, network models of molecular chains which make easy to compose the meso-scale structure, introduced to simulate the meso-scale interactions. Some network models are constructed. These models have different molecular weight distribution and structure. Large strain deformation of these network models is evolved via the FEM analysis. In this calculation, the effects of Molecular chain entanglement, slipping, chain broken, and Van der Waals' force are also considered. Partitioning of the stress calculation indicates that fracture strain, and tensile strength are increase, as molecular weight distribution become wide.

\section{INTRODUCTION}

Recently, the electronics devices become smaller and thinner. The polymers are used for advantage of lightweight and good formability. It is important to understand the mechanical properties of polymers for design of the electronics devices. The mechanical properties of polymers depend on meso-scale structure and interactions such as chain entanglements, orientation, breaking of molecular chains and molecular weight distribution, etc. These meso-scale behaviors are very complex and it is difficult to clarify their influences on the mechanical properties at macro-scale. Various studies related to this problem have been made. De Gennes[1] discuss ed the motions for one chain performing wormlike displacements inside a strongly corss-linked polymer gel. Theodorou and Suter[2] suggested the method for the detailed atomistic modeling of well-relaxed amorphous glassy polymers. Shih and Shih[3] studied semidilute athermal polymer solutions near a hard wall with Monte Carlo simulations. Kho-hei[4] suggested a primitive molecular theory for stress-strain relationship of spherulitic polymers. Using molecular dynamics simulations, Stevens [5] studied the effect of interfacial bond density and network size on interfacial fracture. Hiroo [6], studied the coarse-graining is applied to flexible polymer chains. Yashiro[7] discussed the mechanical properties from atomistic scale. These works have been clarified some aspects of mechanical properties. However, the influence of meso-scale behavior on mechanical properties at macro-scale is still unclear.

In this paper, network models of polymer chain are used to simulate the meso-scale behavior. From the finite element analysis of this model, macro scale mechanical properties, such as stress-stretch ratio curve, are investigated. The effects of the meso-scale structure, especially molecular weight distribution, to macro mechanical properties of polymeric materials are also discussed. 
2.1 Modeling of molecular chain

\section{SIMULATION MODEL}

It is the one of the main characteristic of polymer that the molecular chain length is long, and the length is variety. In this study, polymeric materials are regarded as assembly of polymer chain. This assumption make easy to reduce the computational time and to extend length (meso-scale) and time scales to be simulated. The molecular chain which is the most basically element in this study, is represented by simple model such as the mass-spring model (Fig. 1). N successive atoms are combined into a single mass-spring segment, whose interaction center is defined as the center of mass. We chose n equal to 10 in the present work. The force of spring is written as

$$
\begin{gathered}
L(x)=\frac{d}{d x} \ln \left(\frac{\sin (h x)}{x}\right)=\operatorname{coth} x-\frac{1}{x} \\
f=\left(\frac{k T}{l}\right) L^{-1}\left(\frac{r}{n l}\right)
\end{gathered}
$$

where the components, $\mathrm{k}, \mathrm{T}, \mathrm{l}, \mathrm{r}$, are the Boltzmann constant , absolute temperature, bond length, and the distance between bonded mass pair. $\mathrm{L}^{-1}$ is inverse Langevin function. 2.2 Modeling of Polymeric materials

It is believed that molecular chains of Polymeric materials have many kinds of length, and entangled each other. The complex meso-scale structures are composed by molecular chain, like Figure 2. In this study, the structures are regarded as molecular chain network model (Fig. 3). Small black circulars represent the masses, large black circulars represent the entanglement points, and white circles represent the slip points. The entanglement can be considered (Fig. 4a). The entanglement has possibility to be physically cross-linked (Fig. 4a). The modeling of entanglement is to be combined the two chains at one node, (Fig. 4a). The slip point is not physically cross-linked. Only chain slipping occurs here (Fig.4b). The modeling of slip point is overlapped two segments (Fig. 4b). The resist force of slip is interacted with Pair White nodes (Fig. 4b). In this study, Van der Waals' force (as will hereinafter be described in detail) is used for resist force. Network models of polymer chains are produced as following method. At first, $m$ chains composed of two segments are randomly arranged on the cubic lattice. Then, these chains starts to move under the following conditions: (1) The front of polymer chain must move to it's adjacent point and drag the rest of chains. (2) The direction of the polymers movement is that the density of the segments is highest. (3) If the location, where the polymer chain wants to move, has already occupied, it must move to the direction that the density of segments is the second highest. (4) If all adjacent points have already occupied by another polymers, this polymer chain stays here and does not move in this step. (5) If the front of polymer chain meets another front of polymer chain at the same point, then these polymers are polymerized. (6) If the polymer chain stays at the same point during next two steps, the front and end of this polymer chain are reversed. The generation process of network model is shown Figure 5. The effectual size models are cut from network models for mechanical analysis. The square line represents cut line (Fig. 5).

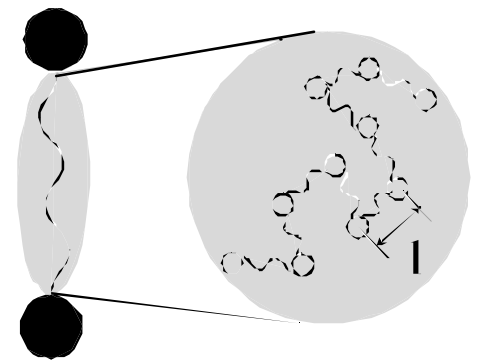

Figure 1. Mass-spring modeling

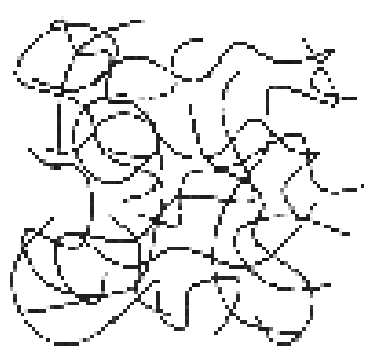

Figure 2. Entangled chains of polymer 


\section{MECHANICAL ANALISYS}

3.1 Chain breaking and Van der Waals' force

The mechanical analysis of molecular chains are simulated by the finite element analysis. In this calculation, the effects of chain entanglement, chain slipping, breaking of molecular chains and Van der Waals' force are also considered. Entanglement and slipping were also explained. The explanation of Chain breaking and Van der Waals' force are follows. The behavior of broken is considered the spring constant is stepped down to 0 gradually. When the segment elongation reaches $\lambda$ times, this molecular chain is assumed to be broken. We chose $\lambda$ equal to 3 in the present work. The intermolecular interactions, in other hands, Van der Waals' force are described by a Lennard-Jones-type potential: Where $\epsilon$ is the characteristic energy well of the potential, $\sigma$ is the excluded volume radius, and $r$ is the distance between two nodes. $\epsilon$ is equal to $1.64 \times$ $10^{-21}[\mathrm{~J}]$ ando is equal to 0.40 [nm] Van der Waals' force affects circle area (Fig. 6), and is used slip point.

\subsection{Relaxation}

The models cut from network models are not in equilibrium and must be relaxed before mechanical analysis. The boundary conditions of the relaxation are Figure 7a, and Figure $7 \mathrm{~b}$ shows the after relaxation model.

3.3 Analysis results

Figure 8 shows the Initial models and boundary condition of deformation. Figure 9 shows the molecular weight fractions. Figures 10 12 depicts the behavior of the models under uniaxial tension. Molecular weight fraction of three models are different, therefore they have different structure too. Model A is separated large concentration parts (center of model A) and large blank part. About model B, large blank areas are divided by molecular chain into small blank. More over, about model C, concentration parts increase. Because of these differences of structures, the deformation shapes are not equal. Figure 10a (model A) show concentration parts are not deformed. But. Lonely chains of blank area are strongly elongated. It is considered the deformation of polymeric material is local deformation. Figure 10b (model B) show the same

$$
\Phi_{L J}=4 \varepsilon\left[\left(\frac{\sigma}{r}\right)^{12}-\left(\frac{\sigma}{r}\right)^{6}\right]
$$

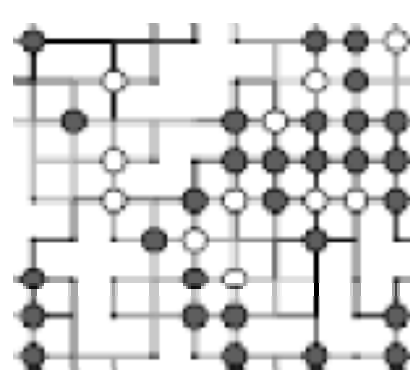

(a) Entanglement point modeling
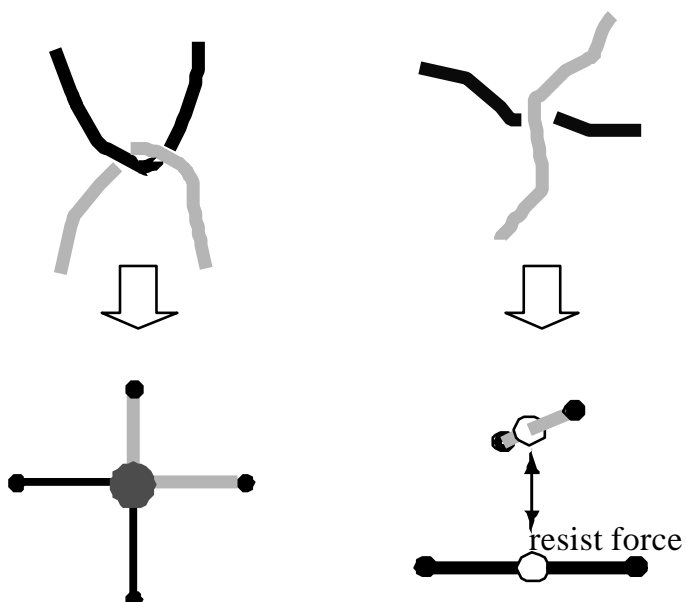

(b) Slip point modeling

Figure. 3 Network model

Figure. 4 Chain configuration and modeling

tendency. However, concentration parts are changed shape small degree. Lonely chains are built up 
other chains. Therefore, not only Lonely chains but also concentration pares are deformed. Figure 11 shows Model A (Fig. 11a) is broken at the first. Because, elongated parts are lonely chains only. On the other hand, concentration parts are also changed shape in model B (Fig.11b), and model C (Fig. 11c). From this reason, fracture strain increase as model A to model B. Figure 12 display the Stress-Stretch ratio curve. The effect of molecular weight distribution is captured. Fracture strain and tensile strength are increased, as molecular weight distribution become wide. That is to say, it is better that chain length becomes long and kind of chain length become variety.

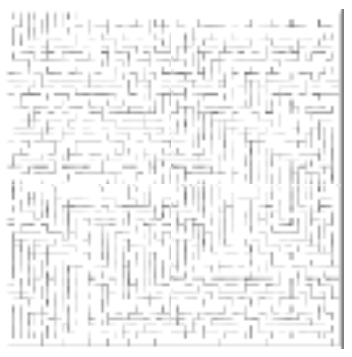

(a) Initial state

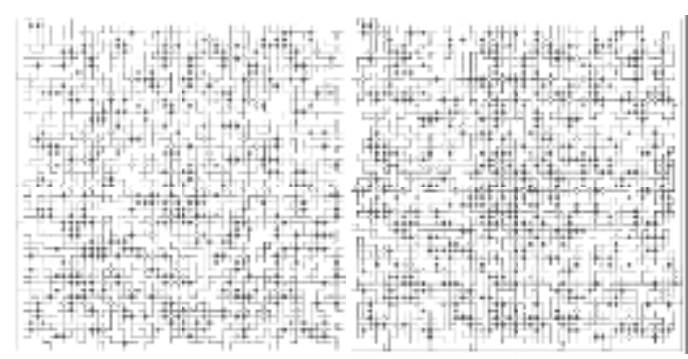

(b) Calculation step 6 (c) Calculation step 10

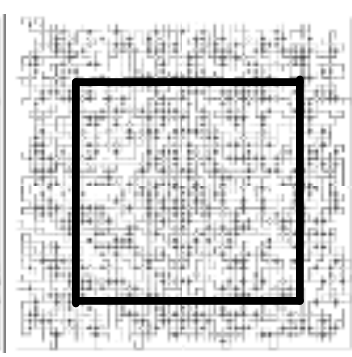

d) Calculation step 20

Figure. 5 Generation process of network model

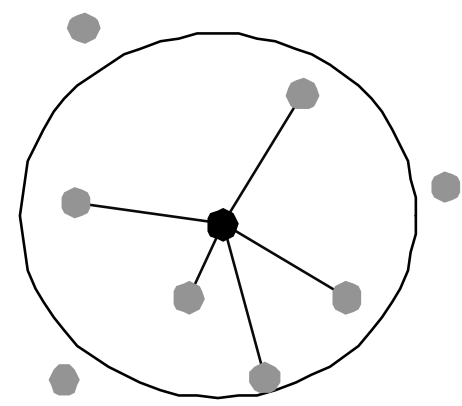

Figure. 6 Efficient area of VDW force

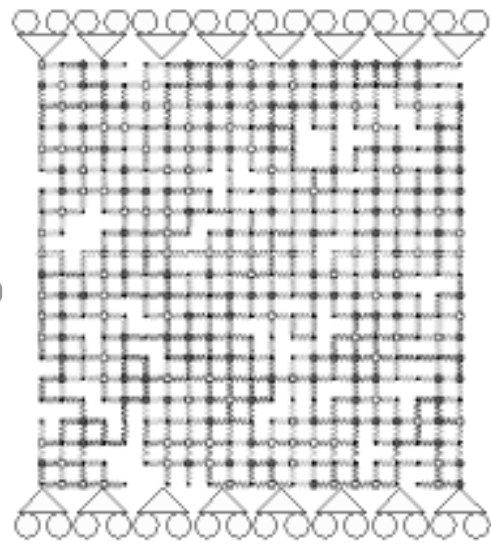

(a) un equilibrium model

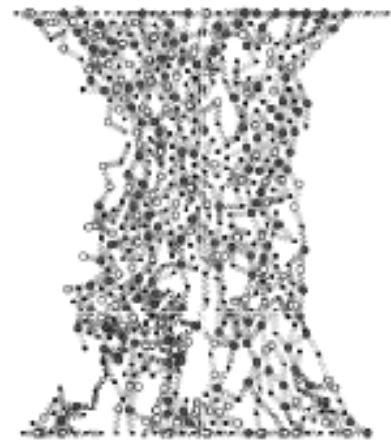

(b) After relaxation model

Figure. 7 Relaxation of net work model

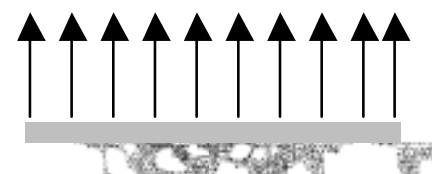

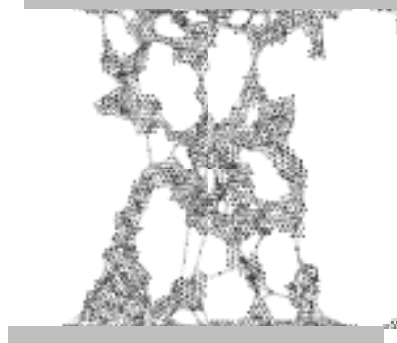

(a) Model A

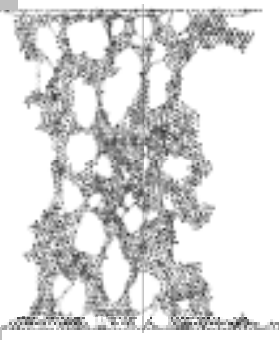

(b) Model B

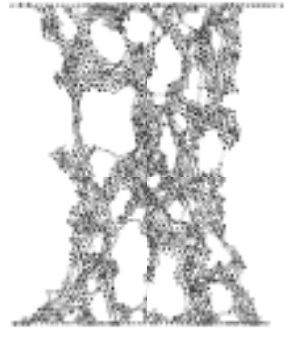

(c) Model C

Figure. 8 Initial states of simulation model 


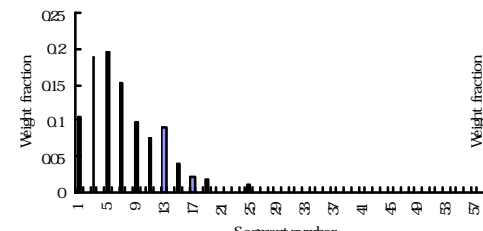

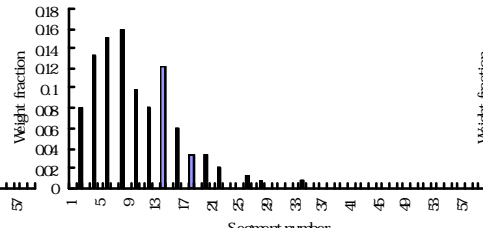

(b) Model B

Figure. 9 Molecular weight distribution

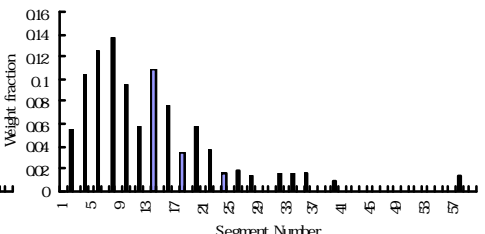

(c) Model C

(a) Model A

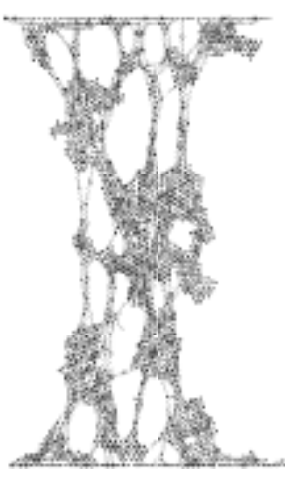

(b) Model B

(a) Model A

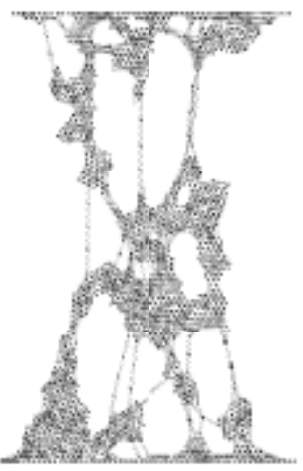

Figure. 10 Deformed states of simulation model (stretch ratio $=1.40$ )

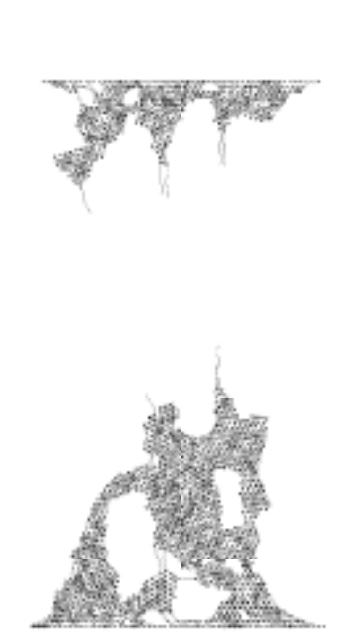

(a) Model A

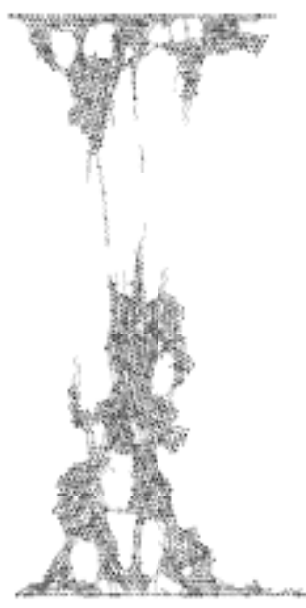

(b) Model B

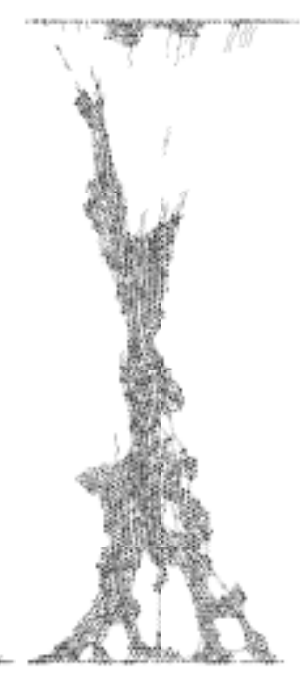

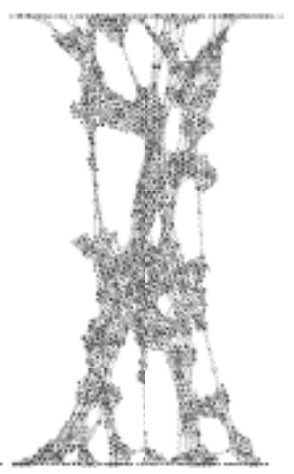

(c) Model C

$\begin{array}{ccc}\text { (a) Model A } & \text { (b) Model B } & \text { (c) Model C } \\ \text { Figure. } 11 \text { Deformed states of simulation model (broken models) }\end{array}$ 


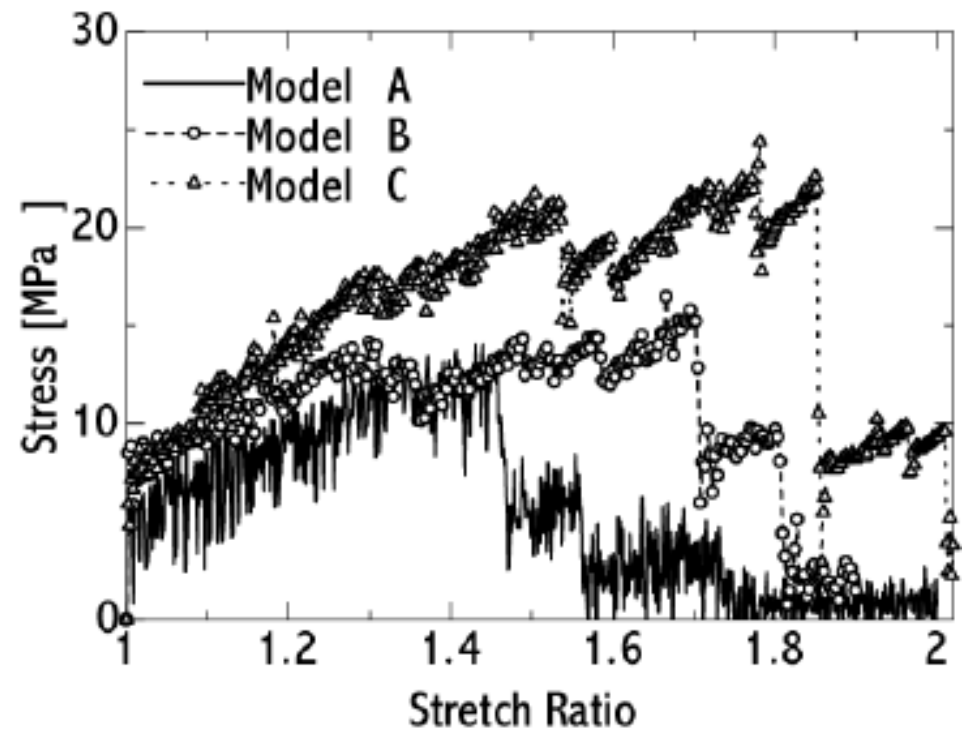

Figure. 12 Stress-stretch ratio curve

\section{CONCLUTION}

The results obtained in this research are summarized as following.

(1) Elongation of the polymers is localized deformation. The deformation shape and mechanical properties are dependent on the meso-scale structure.

(2) Fracture strain and tensile strength are dependent on the molecular weight distribution. They are increased as the molecular weight distribution become wide.

1. P.G. de Gennes, J. Chem. Phys, 55 (1971) p. 572

2. D.N. Theodorou, and U.W. Suter, Macromolecules, 18 (1985) P. 1467

3. W.Y. Shih, W.H. Shin, and I.A. Aksay, Macromolecules, 23 (1990) P. 3291

4. N.Koh-hei, Computational and Theoretical Polymer Science, 9 (1996) P. 19

5. M.J. Stevens, Macromolecules, 34 (2001) p.2710

6. F. Hiroo, T. Jun-ichi, D. Masao, J. Chem. Ohys. 116 (2002) p.8183

7. Y. Kisaragi, I. Tomohiro, T. Yoshihiro, International Journal of Mechanical Sciences 45 (2003) P.1863 